\title{
Use of a Single Coil Transvenous Electrode with an Abdominally Placed Implantable Cardioverter Defibrillator in Children
}

\author{
PETER S. FISCHBACH, IAN H. LAW, MACDONALD DICK II, SARAH LEROY, \\ RALPH S. MOSCA, and GERALD A. SERWER \\ From the University of Michigan Congenital Heart Center, University of Michigan Health System, \\ Ann Arbor, Michigan
}

FISCHBACH, P.S., ET AL.: Use of a Single Coil Transvenous Electrode with an Abdominally Placed Implantable Cardioverter Defibrillator in Children. While transvenous defibrillator electrode placement avoiding a thoracotomy is preferable, electrode size, a large intercoil spacing, and the need for subclavicular device placement preclude this approach in most children. We investigated a single $R V$ coil to an abdominally placed active can ICD device. Five children ages 8-16 years (weight 21-50 kg, mean $35 \mathrm{~kg}$ ) underwent ICD placement. Placement of a single coil Medtronic model 6932 or 6943 electrode was performed via the left subclavian vein approach and the electrode positioned in the RV apex with the coil lying along the RV diaphragmatic surface. The ICD (Medtronic Micro Jewel II model 7223Cx) was implanted in a left abdominal pocket with the lead tunneled from the infraclavicular region to the pocket. Implant DFTs were $\leq 15 \mathrm{~J}$ using a biphasic waveform. DFTs rechecked within 3-month postimplant were unchanged. Lead impedance at implant ranged from 38 to $56 \Omega$, mean $51 \Omega$. Follow-up was 3-21 months (total 82 months) with no electrode dislodgment, lead fractures, or inappropriate discharges. Two of the five patients have had successful appropriate ICD discharges. Transvenous ICD electrode placement can be performed in children as small as $20 \mathrm{~kg}$ with the device implanted in a cosmetically acceptable abdominal pocket that is well tolerated. Excellent DFTs can be achieved. This approach avoids a thoracotomy in all but the smallest child, does not require subclavicular placement of the device, and avoids use of a second intravascular coil. (PACE 2000; 23:884-887)

defibrillator, children, arrhythmia, abdominal implant

\section{Introduction}

The value of implantable cardioverter defibrillators (ICDs) as a lifesaving intervention has been previously demonstrated in the pediatric population. ${ }^{1}$ The experience with ICDs in this age group is limited, accounting for less than $1 \%$ of all implanted devices. ${ }^{1}$ Similar to the indications for device implantation in adult patients as outlined recently by the American College of Cardiology and the American Heart Association, ${ }^{2}$ the need for an ICD in the pediatric population arises from one of three causes: (1) primary electrical disease (congenital long QT syndrome, idiopathic ventricular fibrillation [VF]); (2) cardiomyopathy (arrhythmogenic right ventricular dysplasia, hypertrophic, dilated); and (3) following repair of congenital heart disease (tetralogy of Fallot, transposition of the great arteries). Previously, the size of the transve-

\footnotetext{
Address for reprints: Peter S. Fischbach, M.D., C.S. Mott Children's Hospital, University of Michigan Medical Center, F1310 MCHC/Box 0204, 1500 East Medical Center Drive, Ann Arbor, MI. 48109-0204. Fax: (734) 936-9470; e-mail: petersf@umich.edu

Received August 24, 1999; accepted October 13, 1999.
}

nous electrodes and the long intershocking coil distance have been prohibitively large for transvenous ICD placement in smaller pediatric patients, and, therefore, required epicardial patch electrode placement. New, smaller diameter single coil leads using the device as the second high voltage electrode have been developed, allowing the placement of endocardial systems in smaller patients. ${ }^{3}$ Despite recent reductions in the size of ICDs, they remain unacceptably large for infraclavicular placement in small patients due to cosmetic and comfort concerns. Similar problems have been resolved in pediatric patients (including infants) requiring permanent cardiac pacing by implanting the pacemaker in an abdominal location. Whereas this implant site would accommodate the large device, its location raises the question as to its ability to defibrillate the heart with this new electrode configuration. This article documents the safety and efficacy of an ICD system using a single coil transvenous electrode and the abdominally implanted device as the second high voltage electrode.

\section{Methods}

Five children (Table I), ages 8-16 years and weighing 21-50 kg, underwent ICD placement fol- 
Table I.

Patient Data

\begin{tabular}{|c|c|c|c|c|c|c|c|}
\hline \multirow[b]{2}{*}{ Patient } & \multirow[b]{2}{*}{ DOB } & \multirow[b]{2}{*}{ Weight } & \multirow[b]{2}{*}{ AAl } & \multirow[b]{2}{*}{ Diagnosis } & \multicolumn{2}{|c|}{ Defibrillation Threshold } & \multirow[b]{2}{*}{ TD } \\
\hline & & & & & Implant & Follow-Up & \\
\hline 1 & $12 / 11 / 81$ & 44.1 & 15.9 & $\begin{array}{l}\text { TOF s/p repair, syncope, } \\
\text { inducible VT ( } C L=220 \mathrm{~ms})\end{array}$ & $<5 J$ & $<10 \mathrm{~J}$ & No \\
\hline 2 & $4 / 26 / 87$ & 28.0 & 10.5 & $\begin{array}{l}\text { Noonan's syndrome, HOCM } \\
\text { s/p myomectomy VT/VF } \\
\text { s/p arrest }\end{array}$ & $<10 \mathrm{~J}$ & $<20 J$ & No \\
\hline 3 & $6 / 30 / 89$ & 22.0 & 8.3 & $\begin{array}{l}\text { Structurally } \mathrm{nl} \text { heart VT w/out } \\
\text { of hospital. arrest }\end{array}$ & $<5 \mathrm{~J}$ & $<10 \mathrm{~J}$ & Yes \\
\hline 4 & $2 / 2 / 84$ & 52.0 & 13.8 & LQTS & $<10 \mathrm{~J}$ & $<10 \mathrm{~J}$ & Yes \\
\hline 5 & $6 / 18 / 88$ & 33.4 & 10.8 & HOCM & $<15 \mathrm{~J}$ & $<20 \mathrm{~J}$ & No \\
\hline
\end{tabular}

$\mathrm{DOB}=$ data of birth; $\mathrm{AAI}=$ age at implant (years); $\mathrm{TD}=$ therapy delivered; $\mathrm{TOF}=$ tetralogy of fallot; $\mathrm{CL}=\mathrm{cycle}$ length; $\mathrm{HOCM}=$ hypertrophic obstructive cardiomyopathy; LQTS = long QT syndrome; VT = ventricular tachycardia.

lowing a syncopal event with documented ventricular tachycardia (patients 1-4) or prophylactically in a patient with hypertrophic cardiomyopathy (patient number 5). A single coil Medtronic model 6932 or 6943 electrode (Medtronic Inc., Minneapolis, MN, USA) was placed via a 10 Fr introducer accessing the left subclavian vein. The electrode was positioned in the right ventricular apex so that the coil lay along the right ventricular diaphragmatic surface. The ICD (Medtronic Micro Jewel II model $7223 \mathrm{Cx}$ ) was implanted in the operating room under general anesthesia in a subcutaneous pocket created in the left upper quadrant. The lead $(75-100 \mathrm{~cm})$ was tunneled from the infraclavicular region along the anterior axillary line to the pocket (Fig. 1). Defibrillation thresholds (DFTs) were determined at the time of implant and repeated within 3 months in all patients. The patients were seen in follow-up at 6-month intervals, and their devices were interrogated at each visit.

\section{Results}

At the time of implant, the DFTs ranged from 5 to $15 \mathrm{~J}$ (mean $9 \mathrm{~J}$ ) with no significant changes at 3-month postimplantation retesting (range 10-20 J, mean $14 \mathrm{~J}$ ). Lead impedance measured at implant ranged from 38 to $56 \Omega$ (mean $51 \Omega$ ). Followup for 3-21months (total 82 months) has been free of electrode dislodgment, lead fractures, or inappropriate discharges. To date, two of the five patients have had successful, appropriate ICD discharges. The remaining three patients have had no device discharges and no symptoms suggestive of recurrent tachyarrhythmias

There were no complications related to the placement of the electrode or ICD. The lone system complication occurred in patient 3 who initially underwent placement of a dual coil shocking electrode and was found to have a fracture in the proximal shocking coil that did not affect the sensing capabilities of the lead or the function of the distal coil. Due to the long intershocking coil distance relative to the size of the patient, the

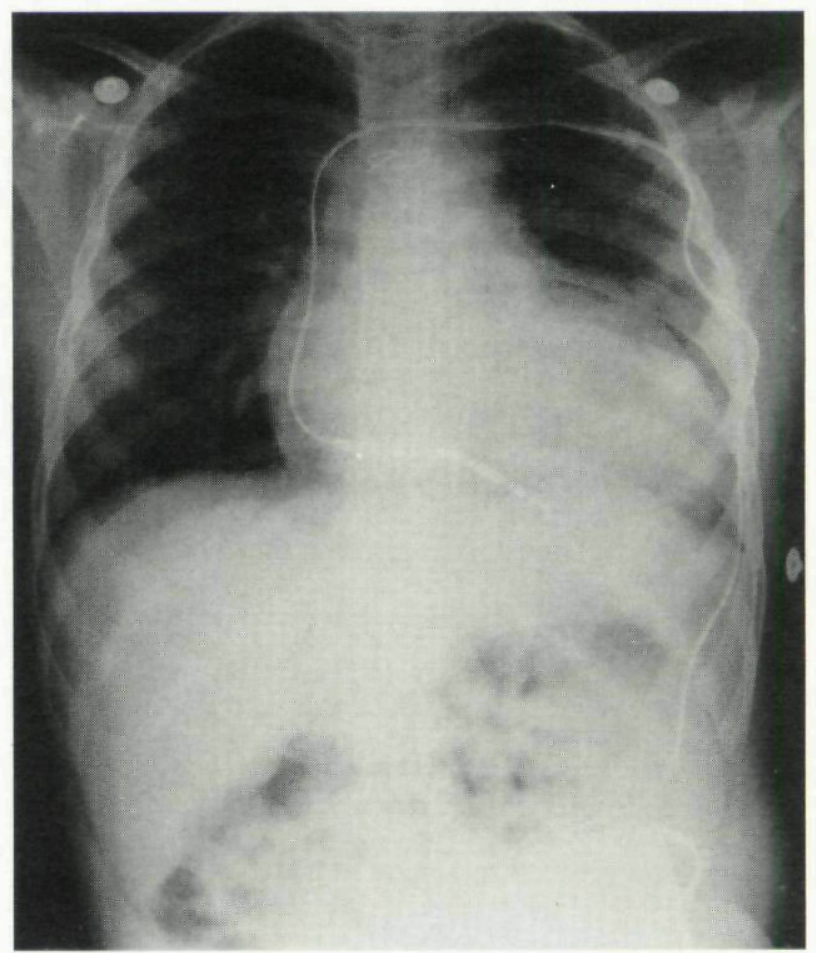

Figure 1. Location of the implantable cardioverter defibrillator (ICD) and course of the defibrillator lead in patient 5. The lead is tunneled from the left infraclavicular region to the left upper abdominal pocket in the operating room. The principle stress points on the lead are where the lead passes between the clavicle and the first rib, and where the lead passes over the edge of the lower costal margin. 

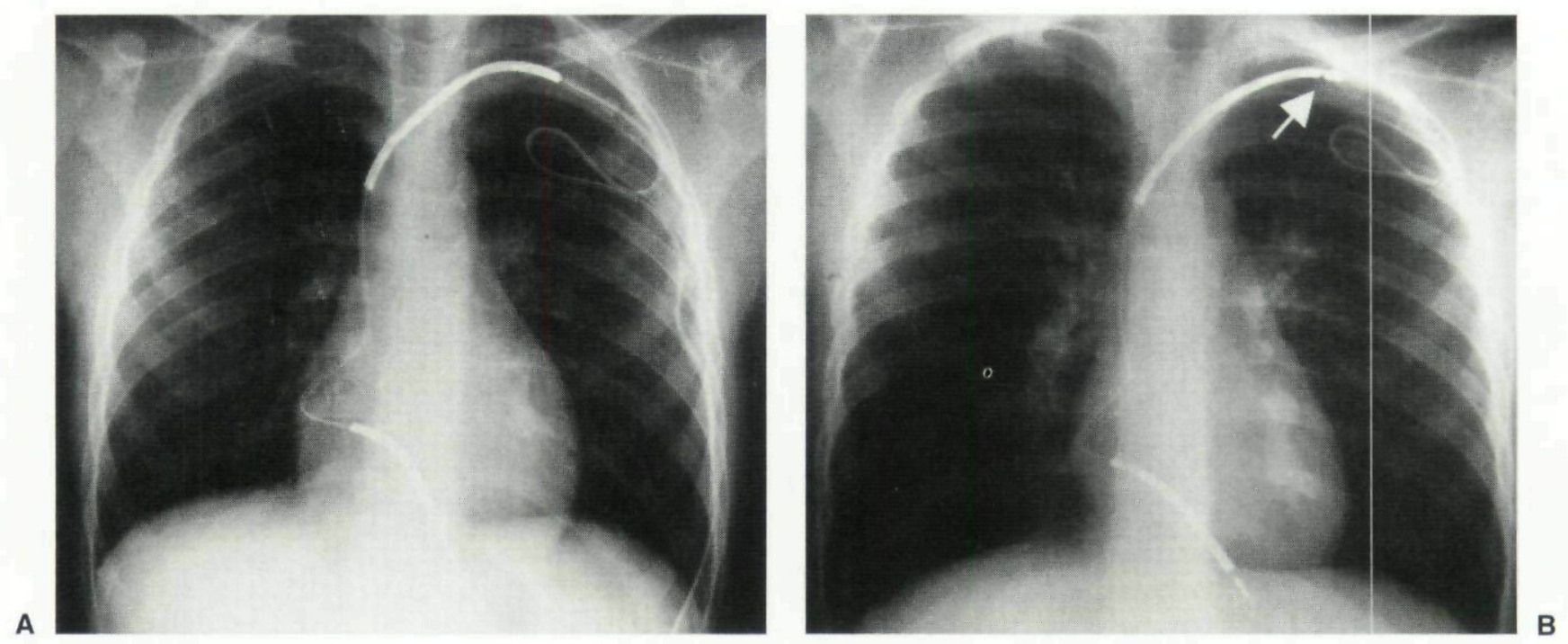

Figure 2. (A) A chest radiograph shortly after the placement of the implantable cardioverter defibrillator (ICD) system in patient 3. Due to the patient's small size relative to the intercoil distance, the proximal coil was left extending beyond the entrance site in the subclavian vein. (B) A subsequent chest radiograph demonstrating a fracture of the proximal high voltage coil as it passes between the clavicle and the first rib (arrow).

proximal coil extended beyond the entry site in the subclavian vein and between the head of the left clavicle and the first rib (Fig. 2). The proximal coil could not be advanced further into the innominate vein at initial implant as this caused dislodgment of the distal electrode in the right ventricle. The device was reprogrammed to shock between the device and the distal coil. The patient had successful appropriate therapies prior to and after the reprogramming of the device.

\section{Discussion}

The implantation of ICDs in pediatric patients is infrequent. However, due to advances in disease recognition, broader acceptance of the device's use in children, and improvements in device technology, their use is increasing. One factor encouraging acceptance of ICDs is the production of smaller devices and endocardial lead systems permitting the placement of transvenous ICDs. These devices eliminate the need for a thoracotomy and its associated morbidity in all but the smallest patients. ${ }^{4}$ While the smallest devices currently available have been reduced to $<40$ cubic centimeters (Medtronic Gem DR, CPI Mini IV), this volume still remains prohibitively large for pectoral implantation in smaller pediatric patients due to comfort and cosmetic reasons. Similar concerns were previously addressed for the implantation of permanent pacing systems in infants and small children. The solution was to place the pulse generator in a pocket in the abdominal wall, which can more easily accommodate the large device relative to the patient's body. This approach has also been used when placing ICD devices in smaller patients using epicardial or dual coil lead systems. The efficacy and safety of an ICD configuration using a single coil intracardiac electrode with an abdominally placed device acting as the second high voltage electrode has not previously been reported.

Zipes and colleagues ${ }^{5}$ first demonstrated that the fibrillating heart requires a minimal myocardial mass be depolarized by an electrical shock to achieve successful defibrillation. One advantage of ICD systems with epicardial defibrillation patches is their anatomic relation to the ventricles directing the defibrillation energy vector across the ventricular myocardium. In a transvenous lead system with the defibrillating pulse passing between two intravascular high voltage coils or between the coils and a pectorally located device, the energy vector is directed anteriorly and superiorly away from the ventricular septum and the left ventricle. As would be expected, transvenous lead systems have higher energy requirements for defibrillation ${ }^{6}$ and ICD implantation frequently was unsuccessful due to these high DFTs. The advent of biphasic defibrillating shock waveforms lessened this problem by lowering the amount of energy required to defibrillate the heart. ${ }^{7.8}$ While the DFTs using a biphasic waveform remain higher in a transvenous system compared with an epicardial system, the difference is not excessive. ${ }^{6}$

With the high voltage electrode configuration used in this study, the energy vector is altered further still. The energy wave is directed inferiorly and anteriorly, further away from the mass of the left ventricle and interventricular septum. There- 


\section{ABDOMINAL ICD PLACEMENT IN CHILDREN}

fore, one concern of this electrode arrangement is that an unacceptable small mass of myocardium would be depolarized at clinically acceptable energy outputs and thus defibrillation rendered less likely. Despite this altered energy vector, we demonstrated that low DFTs were obtainable $(\leq 15$ $\mathrm{J})$ and that these values were stable at follow-up testing. The clinical efficacy of this system has been confirmed by the two patients who have experienced successful appropriate ICD discharges with restoration of sinus rhythm.

Further concerns have been raised in regards to lead durability. The lead is particularly vulnerable as it passes between the clavicle and rib cage, and as it passes over the lower costal margin to the abdominal pocket. With the exception of patient 3 , where the proximal shocking coil was entrapped between the clavicle and the first rib, no problems have been observed with the leads implanted in these five patients. This complication was considered to be a function of the long

\section{References}

1. Silka M, Kron J, Dunnigan A, et al. Sudden cardiac death and the use of implantable cardioverter-defibrillators in pediatric patients. Circulation 1993; 87:800-807

2. Gregoratos G, Cheitlin M, Conill A, et al. ACC/AHA Guidelines for implantation of cardiac pacemakers and antiarrhythmia devices: A report of the American College of Cardiology/American Heart Association task force on practice guidelines (committee on pacemaker implantation). J Am Coll Cardiol 1998; 31:1175-1209.

3. Bardy GH, Johnson G, Poole JE, et al. A simplified, single-lead unipolar transvenous cardioversion-defibrillation system. Circulation $1993 ; 88: 543-547$.

4. Henthorn R, Waller T, Hiratzka L. Are the benefits of the automatic implantable cardioverter-defibrillator (AICD) overestimated by sudden death rate. J Am Coll Cardiol 1991; 17:1593-1594. intercoil distance leaving the larger diameter, less flexible coil in this vulnerable position. While the follow-up in patient years is small in this series, we also have not experienced any lead fractures in a larger cohort of patients with dual coil systems and abdominally implanted devices. Additionally, the system benefits in comparison with an epicardial patch system by having an endocardial sensing lead making the detection and discrimination of malignant ventricular arrhythmias more sensitive.

In summary, we have demonstrated that transvenous ICD placement can be performed in children as small as $20 \mathrm{~kg}$ using a single coil RV electrode with the device implanted in a cosmetically acceptable, well-tolerated abdominal pocket serving as the second high voltage electrode. Low DFTs can be achieved and are maintained at follow-up. This approach avoids a thoracotomy in all but the smallest child, does not require subclavicular placement of the device, and avoids use of a second intravascular coil.

5. Zipes D, Fischer J, King R, et al. Termination of ventricular fibrillation in dogs by depolarizing a critical amount of myocardium. Am Cardiol $1975 ; 36: 37-44$

6. Zipes D, Roberts D. Results of the international study of the implantable pacemaker cardioverter-defibrillator: A comparison of epicardial and endocardial lead systems. Circulation 1995; 92: 59-65.

7. Block M, Hammel D, Bocker D, et al. A prospective randomized cross-over comparison of mono- and biphasic defibrillation using nonthoracotomy lead configurations in humans. J Card Electrophysiol 1994; 5:581-590.

8. Neuzner J, Pitschner HF, Huth C, et al. Effect of biphasic waveform pulse on endocardial defibrillation efficacy in humans. PACE 1994; $17: 207-212$ 
This document is a scanned copy of a printed document. No warranty is given about the accuracy of the copy. Users should refer to the original published version of the material. 